Received: 20 March 2018

Accepted: 22 June 2018

Published online: 05 July 2018

\section{Contactless Fluid Manipulation in Air: Droplet Coalescence and Active Mixing by Acoustic Levitation}

\author{
Ayumu Watanabe $^{1}$, Koji Hasegawa ${ }^{2}$ \& Yutaka Abe ${ }^{3}$
}

Acoustic manipulation by an ultrasonic phased array provides an entirely new approach to processes such as coalescence, mixing, separation, and evaporation occurring in the generation of new materials, physical property measurement, the biomedical industry, etc. However, to date, ultrasonic phased arrays have not been fully investigated for applications in fluid manipulation. This paper provides contactless coalescence and mixing techniques for droplets in air by controlling the acoustic potential by using an ultrasonic phased array. We focused on mode oscillation to propose an efficient mixing technique for liquid without contact. A comparison of mixing performance between cases with mode oscillation and without mode oscillation showed that the flow induced by mode oscillation promotes droplet mixing. Our paper demonstrates the feasibility of contactless coalescence and mixing as a first step in fluid manipulation with a phased array.

Levitation technologies have recently been examined for utilization in container-less processing ${ }^{1-4}$. Because of its usefulness as a tool for contactless manipulation of fluids, acoustic levitation has been explored in the fields of materials science, analytical chemistry, and biomedicine ${ }^{5-10}$. Several approaches have been investigated for the contactless handling of matter in air. Foresti et al. ${ }^{11}$ enabled the transport and coalescence of acoustically levitated droplets by controlling the vibration velocity amplitude of each emitting element arrayed in a row. Ochiai et al. ${ }^{12}$ developed an acoustic manipulation device by using focused ultrasound transmitted from an ultrasonic phased array and enabled three-dimensional transport of solid particles by controlling the phases of transducers. Marzo et al..$^{13}$ proposed a technique for freely forming arbitrary sound fields by incorporating the appropriate phase differences into an ultrasonic phased array. Nonlinear and dynamic behavior (e.g., internal and external flow of levitated droplets ${ }^{14-18}$, interfacial deformation and atomization ${ }^{19-22}$ ) arise in droplet manipulation by acoustic levitation. Acoustic streaming ${ }^{23}$ is known to be caused by a result of some acoustic energy being converted to a driving force for moving fluid. In a strong sound field in which nonlinear phenomena are observed, a droplet transforms from a sphere into an ellipsoid and is atomized ${ }^{24}$.

Although an ultrasonic phased array enables high precision and free generation of sound fields, most studies use solid particles, which are easy to handle, and an ultrasonic phased array is not applied for droplet manipulation. Our objective was to provide an entirely new approach for contactless fluid manipulation in air to support processes such as coalescence, mixing, separation, and evaporation. In this paper, we present a fundamental technology for levitation, coalescence and active mixing of droplets by using an ultrasonic phased array.

\section{Results}

Acoustic levitation by ultrasonic phased array. The acoustic manipulator is based on an ultrasonic phased array for high precision and free generation of a sound field. This device is composed of compact transducers arranged in a rectangular shape. By controlling the phase of the signal applied to each transducer, the sound waves transmitted from the transducers are focused to one point in space. The reflector faces the phased array, and the focal point of the sound generated is formed on the reflector surface. Focused ultrasound is reflected, and a localized standing wave is generated around the focal point.

A sound field was analyzed by the distributed point source method (DPSM) $)^{25,26}$. Details of the DPSM are described in supplementary information. Figure la shows the calculation results for the case in which a single focal point was formed at the intersection of the central axis of the phased array and the reflector surface. A

${ }^{1}$ Graduate School of Systems and Information Engineering, University of Tsukuba, Tsukuba, Japan. ${ }^{2}$ Department of Mechanical Engineering, Kogakuin University, Tokyo, Japan. ${ }^{3}$ Faculty of Engineering, Information and Systems, University of Tsukuba, Tsukuba, Japan. Correspondence and requests for materials should be addressed to A.W. (email: awatanabelab@gmail.com) 


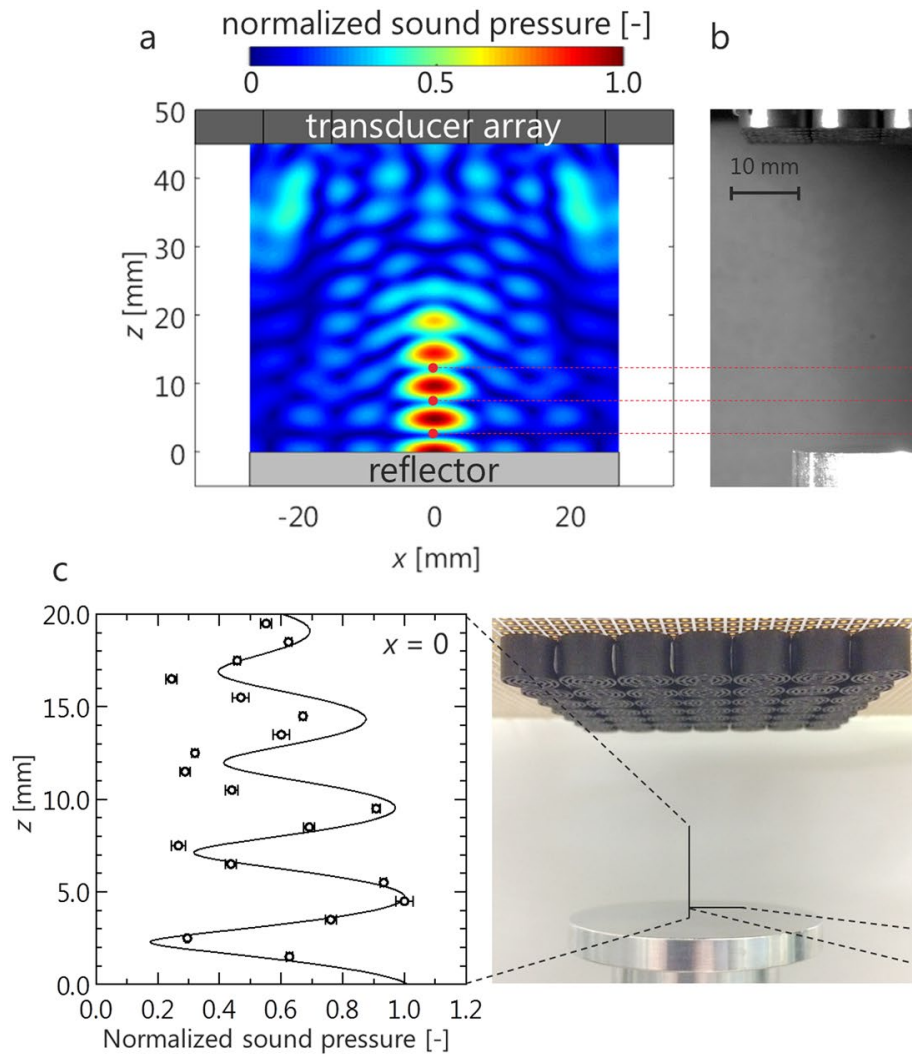

b

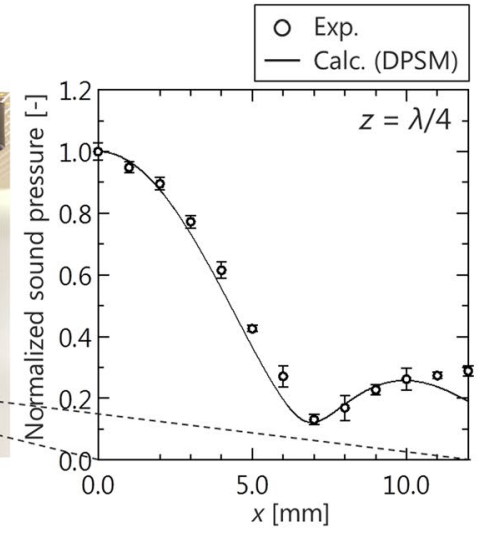

Figure 1. Acoustic levitation by an ultrasonic phased array. (a) Calculation result of acoustic field generated by focused ultrasound. (b) Snapshot of acoustically levitated droplets in localized standing wave. (c) Comparison of sound pressure between experiment and calculation (DPSM). The error bars in the experimental plot represent standard deviations.

high-pressure region was generated in a narrow area near the focal point, and the droplet was expected to be trapped in a limited area near the focal point. A snapshot of the droplets formed near the focal point is shown in Fig. 1b. Ultimately, levitation of the droplets in the localized standing wave was demonstrated. The sound pressure distribution measured by a microphone and that calculated by the DPSM are compared in Fig. 1c. These pressures are normalized by the maximum pressure. Here, $\lambda$ is the sound wavelength of $8.5 \mathrm{~mm}$. For the central axis $(x=y=0)$, the tendency of the sound pressure to decrease with distance from the focal point was demonstrated by both the experimental and calculation results. For the case of a pressure antinode $(z=\lambda / 4, y=0)$, a distribution similar to the shape of a sinc function was indicated by both the experimental and calculation results. The distribution is theoretically derived based on the sound field generated by a rectangular phased array on the focal plane, which follows a sinc function ${ }^{27}$. This tendency was indicated by the experimental results and was reproduced by the calculation results. Therefore, the sound field generated by the phased array system can be reproduced by DPSM calculations.

Contactless coalescence of acoustically levitated droplets by using an ultrasonic phased array. The contactless coalescence requires the generation of two focal points. Focal points were generated artificially by rapidly switching two focal points. A previous study reported that two particles were successfully levitated by switching two focal points at $500 \mathrm{~Hz}^{28}$. We therefore selected the switching frequency to be $500 \mathrm{~Hz}$.

To determine the levitation points based on the acoustic radiation force acting on levitated matter, the acoustic potential was calculated. The Gor'kov potential ${ }^{29}$ was defined as a field, the gradient of which gives the acoustic radiation force acting on a small sphere in a sound field:

$$
U=2 \pi R^{3}\left(\frac{p_{\mathrm{rms}}^{2}}{3 \rho_{\mathrm{G}} c^{2}}-\frac{\rho_{\mathrm{G}} v_{\mathrm{rms}}^{2}}{2}\right),
$$

where $R$ is the radius of a small sphere, $\rho_{\mathrm{G}}$ is the density of air, $c$ is the speed of sound, and $p_{\mathrm{rms}}$ and $v_{\mathrm{rms}}$ are the root mean square value of the sound pressure and particle velocity, respectively. To determine the acoustic potential, the sound pressure and particle velocity were calculated using the DPSM. Figure 2a shows the calculation results for an acoustic potential. When the spacing between the left and right focal points $L$ was $12 \mathrm{~mm}$ (Fig. 2a-1), localized standing waves were generated at each focal point. As the distance between the focal points was reduced (Fig. 2a-2-a-4), the two standing waves formed one large standing wave. For a more detailed study, the acoustic potential at the levitation height $(z=3 \lambda / 4)$ was calculated, as shown in Fig. 2b. At $L=12 \mathrm{~mm}$ (Fig. 2b-1), a 


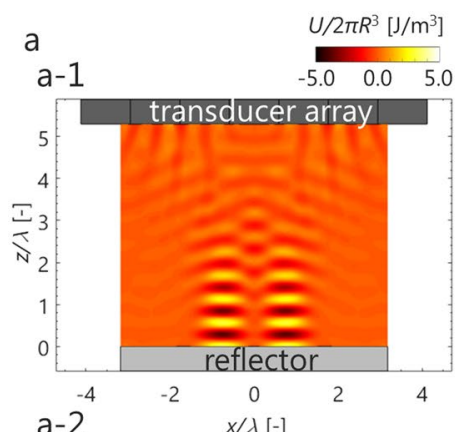

b c

C

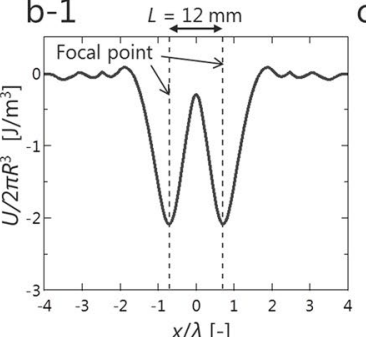

\section{c-1}

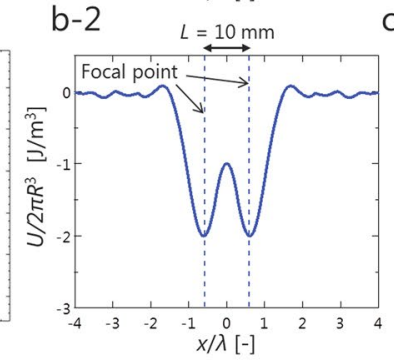

c-2
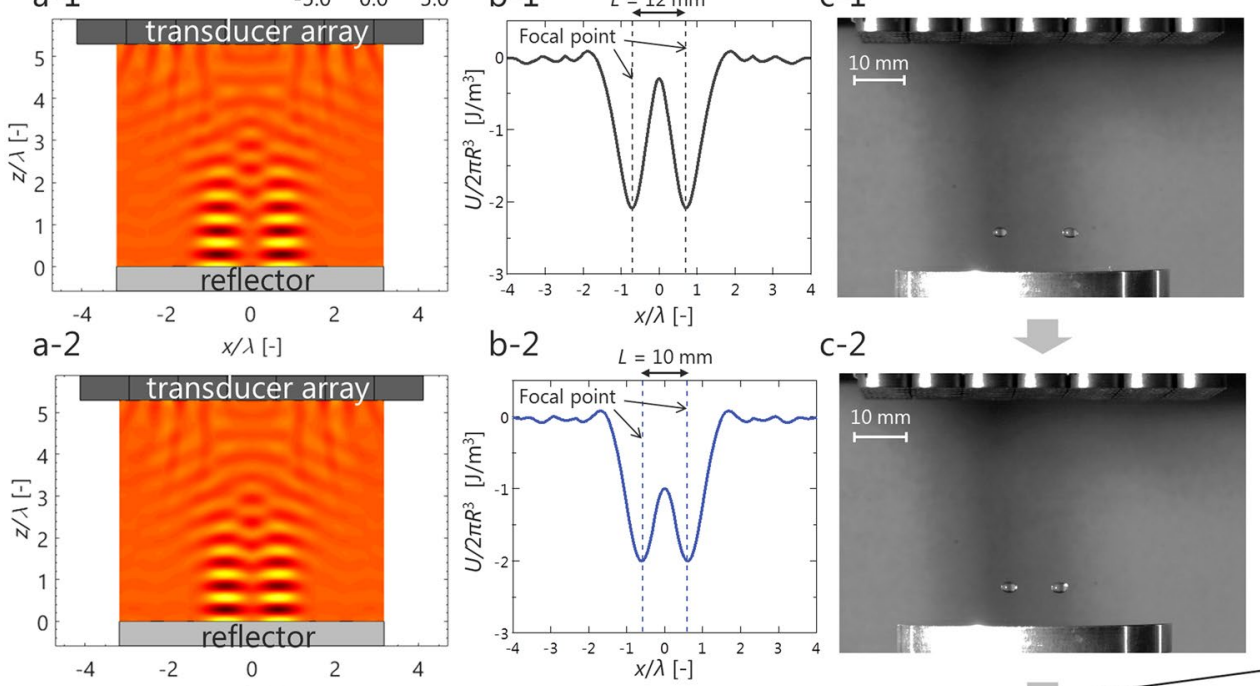

d
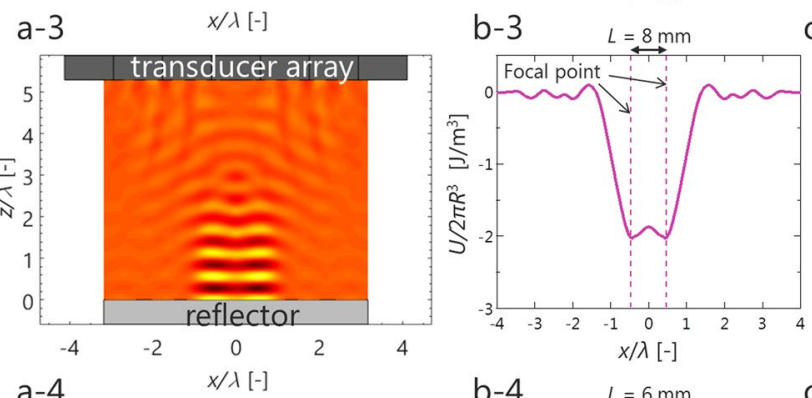

c-3
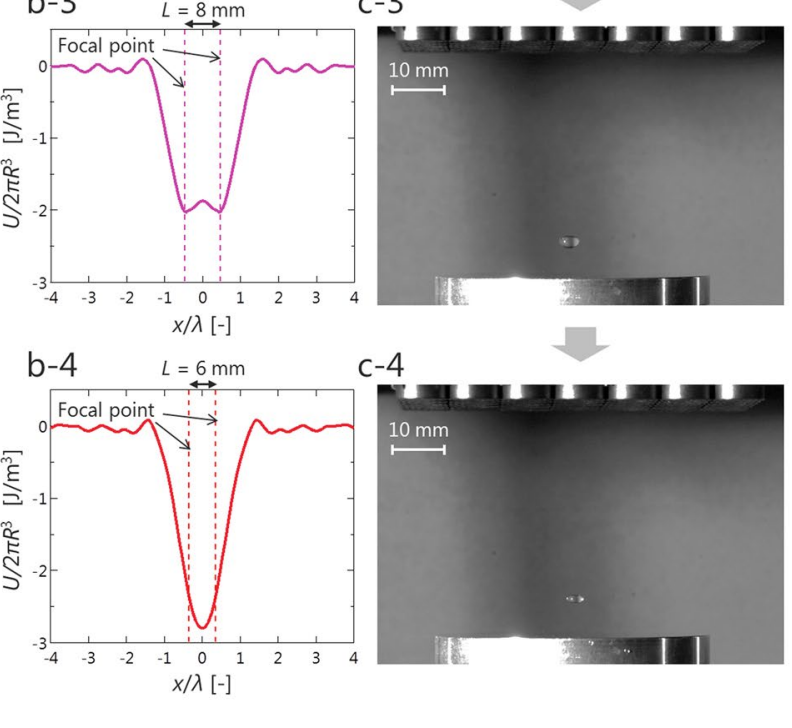

C-4
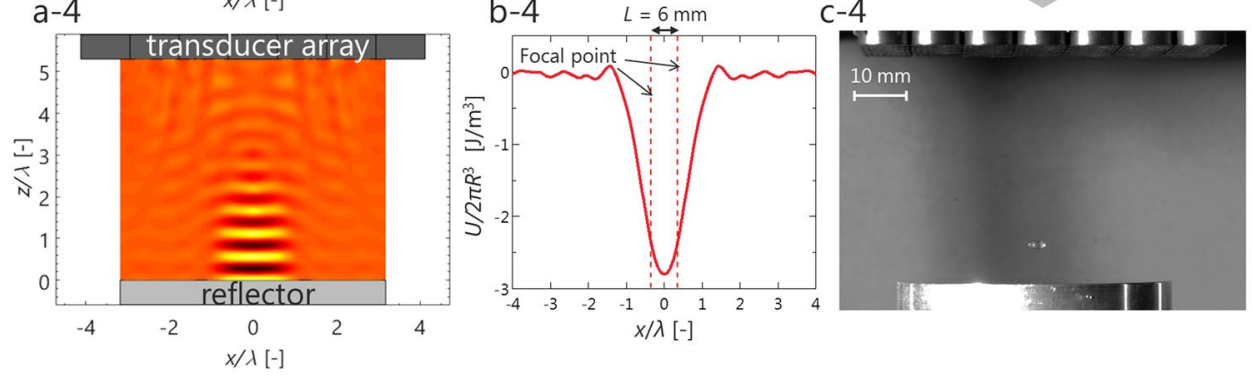

Figure 2. Contactless transport and coalescence by controlling the acoustic potential. (a) Calculation results of an acoustic potential obtained by DPSM (a-1, $L=12 \mathrm{~mm}$; $\mathrm{a}-2, L=10 \mathrm{~mm} ; \mathrm{a}-3, L=8 \mathrm{~mm} ; \mathrm{a}-4, L=6 \mathrm{~mm})$. (b) Calculation results of an acoustic potential at a pressure node $(z=3 \lambda / 4)(\mathrm{b}-1, L=12 \mathrm{~mm}$; b-2, $L=10 \mathrm{~mm}$; b-3, $L=8 \mathrm{~mm}$; b-4, $L=6 \mathrm{~mm}$ ). (c) Snapshots of levitation behavior when a pair of focal points is generated (c-1, $L=12 \mathrm{~mm} ; \mathrm{c}-2, L=10 \mathrm{~mm}$; $-3, L=8 \mathrm{~mm} ; \mathrm{c}-4, L=6 \mathrm{~mm}$ ). (d) Snapshot of contactless coalescence of water droplets.

distinct acoustic potential well was generated at each focal point. At $L=10 \mathrm{~mm}$ (Fig. 2b-2), although the potential distribution shifted to that of a single well, two potential wells still existed at each focal point. This result is consistent with the experimental result shown in Fig. 2c-1, c-2. At $L=8 \mathrm{~mm}$ (Fig. 2b-3), the potential shape transitioned from a two shape to a single large one. This result suggests that each droplet experienced a driving force toward the center. This result is consistent with the experimental result shown Fig. 2c-3,c-4. Figure $2 \mathrm{~d}$ and Supplementary video $S 1$ shows the behavior observed when $L$ was switched from $10 \mathrm{~mm}$ to $8 \mathrm{~mm}$. This result demonstrates the feasibility of contactless coalescence of acoustically levitated droplets using an ultrasonic phased array.

Contactless mixing with resonant oscillation. Active mixing techniques in air are effective for biomedical applications. Our work provides a contactless mixing technique with interface oscillation. Shen et al. ${ }^{30}$ reported that the oscillation mode of acoustically levitated droplets was induced by amplitude modulation of ultrasonic waves. The same method was used in this study. To apply oscillation to droplets without contact, the voltage applied to the transducers was modulated by 0 to 1 square wave. The modulation frequency can be tuned in $1 \mathrm{~Hz}$ increments. The typical oscillation behavior is shown in Fig. 3a; the figure shows bottom-view images. The test fluid was $2 \mathrm{cSt}$ silicone oil. Modes were determined by the number of protrusions and then classified into the $4^{\text {th }}$ to $7^{\text {th }}$ mode. The oscillation frequency of droplets coincided within $\pm 1 \%$ to half of the frequency of the 
a
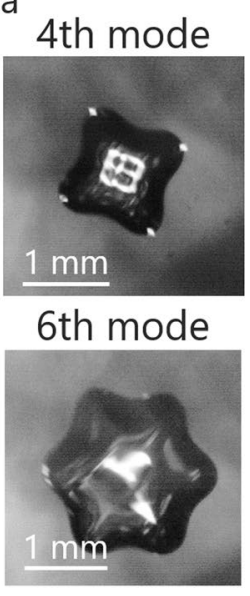

5th mode

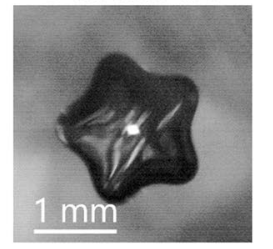

7th mode
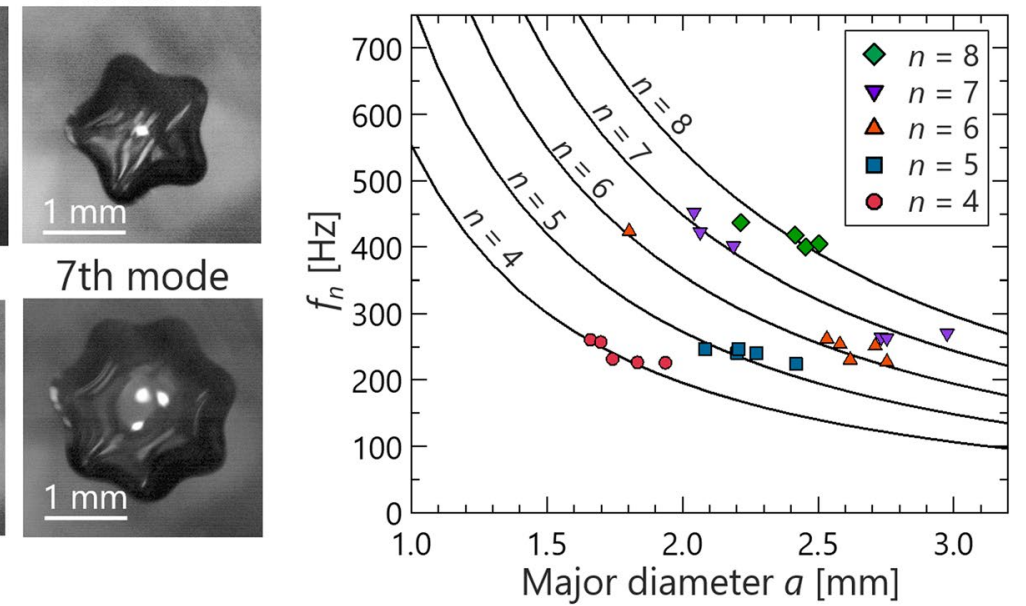

Figure 3. Induction of an oscillation mode by modulation of sound. (a) Typical $4^{\text {th }}$ to $7^{\text {th }}$ mode behavior of acoustically levitated droplet. (b) Condition under which oscillation mode appears. The symbols are the experimental data for the $2^{\text {nd }}$ to $8^{\text {th }}$ mode. Solid curves are the calculation results obtained using Eq. (2).

modulation. Therefore, it is considered that the oscillation mechanism was parametric resonance, as described by Shen et al. ${ }^{30}$. To control the oscillation mode, the conditions under which the mode appears were investigated and summarized in Fig. $3 \mathrm{~b}$ by measuring the relationship between the resonant frequency and the droplet diameter. The solid curve is described by replacing the diameter of the Rayleigh equation ${ }^{31}$ with the major diameter:

$$
f_{n}=\frac{1}{2 \pi} \sqrt{\frac{8 \sigma_{\mathrm{L}}}{\rho_{\mathrm{L}} a^{3}} n(n-1)(n+2)}
$$

where $\sigma_{\mathrm{L}}$ is surface tension, $\rho_{\mathrm{L}}$ is the density of a droplet, $a$ is the major diameter, and $n$ is the oscillation mode. The theoretical value yielded by eq. (2) is reflected in the experimental results. Therefore, the Rayleigh equation, assuming a spherical droplet, can be extended to an acoustically levitated droplet by adopting the major diameter as the diameter. This finding agrees well with Shen's result ${ }^{30}$ and shows that an oscillation mode is governed by the Rayleigh equation.

Differences in mixing behavior between the case with a mode and the case without a mode were investigated by laser-induced fluorescence (LIF). A $50 \mathrm{wt} \%$ glycerin aqueous solution was used for the observation of mixing behavior. Because the fluctuations of a viscous droplet after collision can be suppressed as much as possible, it is expected that the observation of mixing behavior is clear. The measurement procedure is shown in Fig. 4a. A droplet containing a fluorescent dye and a droplet without the dye coalesced and were levitated for 5 seconds to remove the disturbance caused by coalescence. The droplets were irradiated from one side with a Nd:YAG sheet laser, and fluorescence emission was observed via a high-speed video camera from the bottom. The major diameter after coalescence was adjusted to approximately $3.9 \mathrm{~mm}$. According to the Rayleigh eq. (2), modes are not induced at an oscillation frequency of $500 \mathrm{~Hz}$ at this diameter, but the $6^{\text {th }}$ mode is induced at an oscillation frequency of $450 \mathrm{~Hz}$. Therefore, we compare the cases with oscillation frequencies $450 \mathrm{~Hz}$ and $500 \mathrm{~Hz}$ after coalescence for 10 seconds.

Figure $4 \mathrm{~b}$ shows the observation results pertaining to mixing behavior. In the case without a mode, the luminance distribution became uniform 60 seconds after coalescence (Supplementary video S2). On the other hand, in the case with the $6^{\text {th }}$ mode, the luminance become uniform within 10 seconds after the mode appeared (Supplementary video S3). The mixed state was evaluated by the mixing parameter $\eta^{32}$. Based on the LIF results, the average $\mu$ and the standard deviation $\sigma$ of the luminance in the $N$ pixels mixed region were calculated. One standard deviation indicates the difference from the fully mixed state. The mixing parameter $\eta$ is defined by using the normalized standard deviation:

$$
\eta=\frac{\left(\frac{\sigma}{\mu}\right)_{t}-\left(\frac{\sigma}{\mu}\right)_{t=\infty}}{\left(\frac{\sigma}{\mu}\right)_{t=0}-\left(\frac{\sigma}{\mu}\right)_{t=\infty}} .
$$

The mixing parameter begins from $\eta=1$ and approaches $\eta=0$ as mixing progresses. The measurement result of the time trace of $\eta$ is shown in Fig. 4c. The image obtained when a droplet collided was used for $t=0$, and the image obtained after 10 minutes-which confirmed that mixing was completed by observation-was used for $t=\infty$. In the case without an oscillation mode, $\eta$ converged to zero after approximately 60 seconds. On the other hand, $\eta$ converged to zero after 15 seconds, that is, within 10 seconds after the mode appeared in the case with the $6^{\text {th }}$ mode. These results show that mixing of droplets can be promoted without contact by using an oscillation mode. 


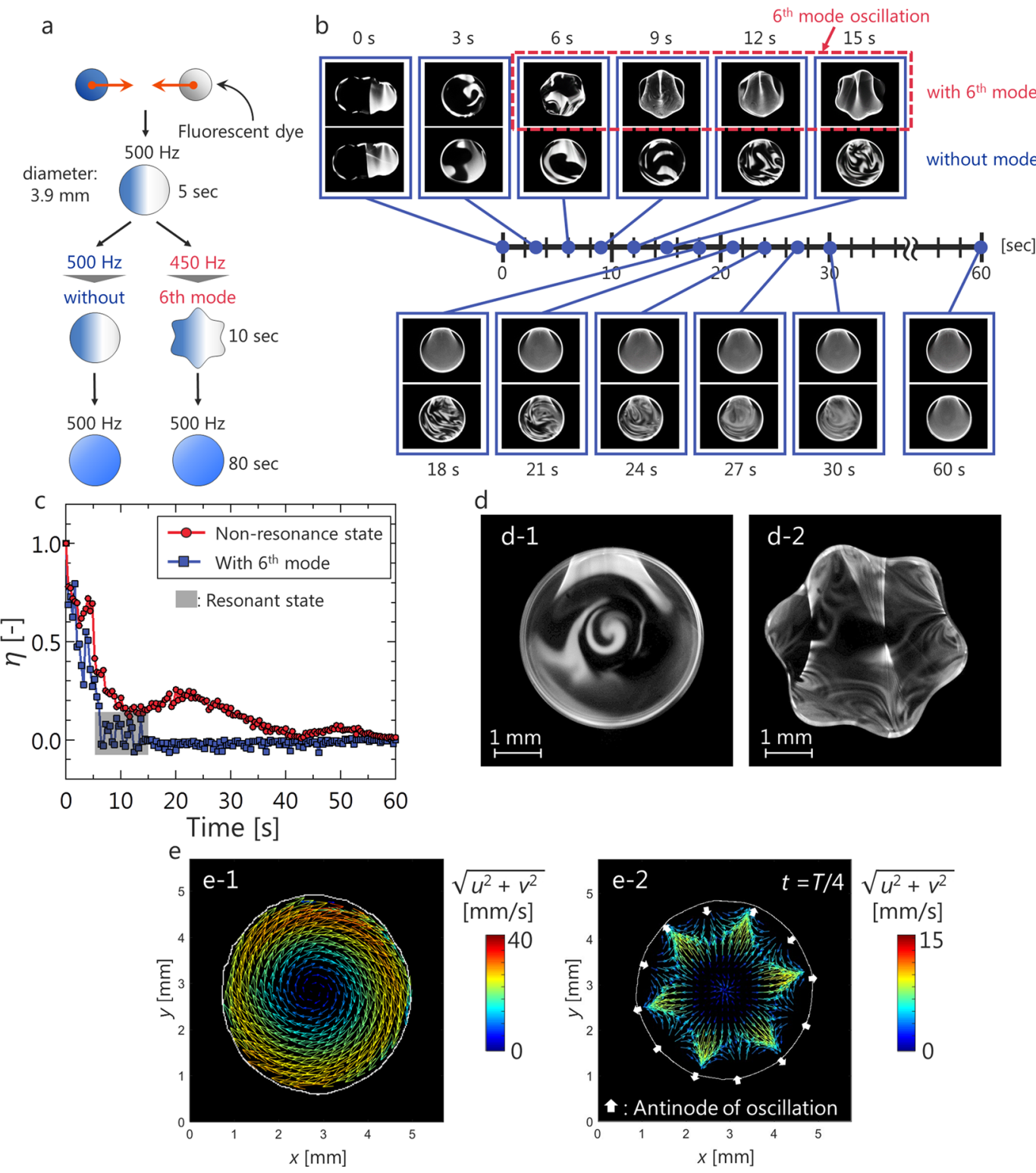

Figure 4. Active mixing of an acoustically levitated droplet by an oscillation mode. (a) Experimental procedure for observing mixing behavior. (b) Comparison of mixing performance between the case without a mode and the case with a mode. (c) Comparison of transition of mixing parameter. (d) Comparison of mixing pattern between (d-1) the case without an oscillation mode and (d-2) the case with an oscillation mode. (e) Comparison of flow structure between (e-1) the case without an oscillation mode and (e-2) the case with a $6^{\text {th }}$-mode oscillation.

A characteristic mixing pattern is shown in Fig. 4d. In the case without a mode (Fig. 4d-1), the mixing pattern showed a swirling characteristic at the center of the droplet. In the case with an oscillation mode (Fig. 4d-2), a vortex-like pattern was formed at the antinode of the oscillation. To investigate why the mixing behavior varied, the internal flow structures of droplets were compared by particle image velocimetry (PIV). A 50 wt\% glycerin aqueous solution was used as the test fluid. The droplets were irradiated from one side by a Nd:YAG sheet laser, and the fluorescence emission of the particles was observed via a high-speed video camera from the bottom. Figure $4 \mathrm{e}-1$ shows the PIV result for the case without oscillation. Rotational flow around one axis occurred inside the droplet. This is the same flow structure found in acoustically levitated glycerol droplets ${ }^{15}$. Figure $4 \mathrm{e}-2$ shows the PIV result for the case with the $6^{\text {th }}$ mode. To clearly observe the flow induced by interface oscillation, the rotational component of the droplet was removed based on the rotational speed measured by tracking the antinode of the oscillation. The white arrow in Fig. 4e B indicates the position of the oscillation antinode and the movement direction of the interface. Near the oscillation antinode, flow occurred toward the same direction of the interface displacement. It was clarified that flow different from that in the non-oscillation state was induced by interface oscillation. 
a

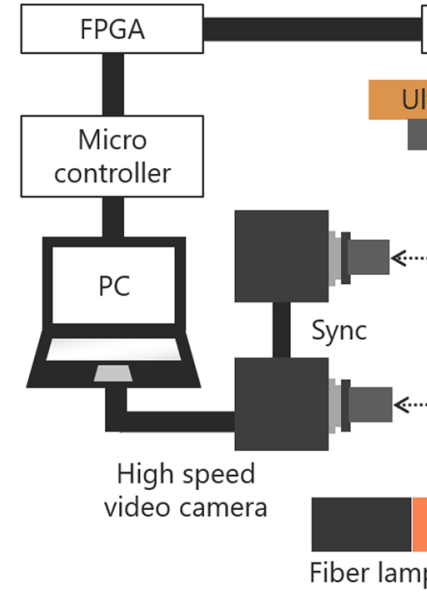

\section{Amplifier \\ Itrasonic phased array}

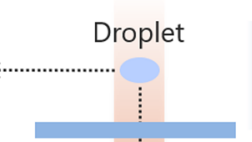

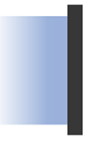

LED light

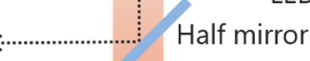

b

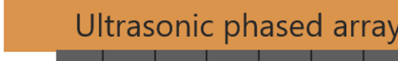

Droplets Fluorescent dye

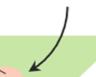

Glass plate

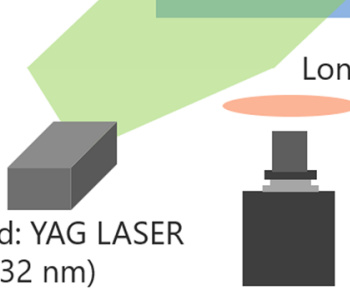

Long pass filter

High speed

video camera
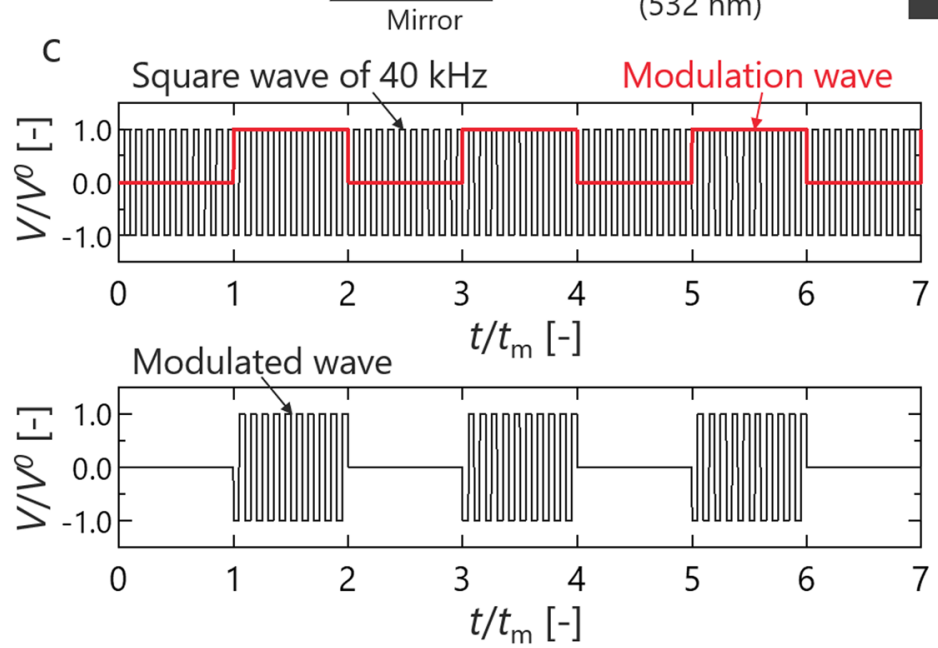

Figure 5. Schematic diagram of experimental apparatus. (a) The experimental system for observing droplet behavior. (b) The experimental PIV and LIF system. (c) Modulation of the voltage applied to the transducers. $V$ is the voltage, $V_{\mathrm{o}}$ is the voltage amplitude, and $T_{\mathrm{m}}$ is the modulation period.

\begin{tabular}{|l|l|l|l|}
\hline Sample & $\rho_{\mathrm{L}}\left(\mathrm{kg} / \mathbf{m}^{3}\right)$ & $\boldsymbol{\mu}_{\mathrm{L}}\left(\mathbf{1 0}^{-\mathbf{3}} \mathbf{P a} \cdot \mathbf{s}\right)$ & $\sigma_{L}(\mathrm{mN} / \mathbf{m})$ \\
\hline Water & 998 & 1.00 & 72.7 \\
\hline 2 cSt Silicone oil (Shin-Etsu Chemical Co., KF-96) & 873 & 1.75 & 18.3 \\
\hline 50 wt\% Glycerin aqueous solution & 1125 & 6.03 & 70.0 \\
\hline
\end{tabular}

Table 1. Properties of test fluids ${ }^{35}$.

\section{Discussion}

A pair of droplets was successfully levitated by generating two acoustic potential wells by controlling the phase of sound. Based on potential estimation (Fig. 2b), it was clarified that the potential shape changed from a two shape to a single large shape by decreasing the gap between the two focal points, and the levitated droplets then moved toward the center and eventually coalesced. It is considered that the conditions under which the droplets coalesce can be predicted by the Gor'kov potential. In this report, although the phase difference at the arbitrary point where sound is focused (the value was calculated based on the geometrical arrangement and speed of sound) was used, by applying the optimal acoustic trap proposed by Marzo et al. ${ }^{13}$, further innovations such as three-dimensional manipulation, giving droplets an angular momentum, and adjustment of the retention force and driving force are expected.

After coalescence, the droplets could be mixed by the oscillation mode. Based on the visualization results pertaining to mixing behavior (Fig. 4), the fluorescence dye inside the droplet was homogenized within 10 seconds after the oscillation mode appeared and in approximately 60 seconds without an oscillation mode. Here, we compared the order of diffusion mixing and convection mixing. The diffusion coefficient ${ }^{33}$ was roughly $D \sim$ $10^{-4} \mathrm{~mm}^{2} / \mathrm{s}$, and the characteristic distance between the droplets was $l \sim 10^{0} \mathrm{~mm}$; thus, the characteristic time of diffusion was $t_{\theta} \sim l^{2} / D \sim 10^{4}$ seconds. Consequently, regardless of the oscillation mode, convection is more 
dominant than diffusion. For the case without an oscillation mode, it is considered that the inertia of coalescence and acoustic streaming inside the droplets promoted mixing. On the other hand, for the case with an oscillation mode, mixing behavior changed drastically by inducing the oscillation mode. Observation results showed that when an oscillation mode appeared, near the antinode of an oscillating droplet, stretching and folding of the interface by the action of a flow ${ }^{34}$ was observed, and it progressed toward the inside of the droplet. It is considered that the formation of vortices via stretching and folding induced by oscillation promotes mixing.

These results offer the potential of creating a platform for contactless fluid manipulation technologies and fundamental fluid science, including not only applied approaches to engineering but also database creation with respect to droplet dynamics. Future studies should address the control over the size of injected droplets and the handling of microscale droplets for biomedical, lab-on-a-drop, and other applications. These fields also require expansion of the technology toward handling processes, including separation and phase change.

\section{Methods}

Experimental setup. Acoustic levitation is achieved by a standing wave at the focal point of sound. By transmitting sound waves with a controlled phase, the focal point of sound is generated at an arbitrary position. By reflecting the focused ultrasound using a reflector, a localized standing wave can be generated near the focal point.

We used a $7 \times 7$ square transducer array consisting of 49 small ultrasonic transducers. The diameter of transducer was $10 \mathrm{~mm}$, the frequency was $40 \mathrm{kHz}$. Phase control of the sound transmitted from each transducer is required to generate an ultrasonic focal point. We realized this control using a field programmable gate array (FPGA) (Altera Co., Cyclone-IV DE0-Nano). The experimental apparatus is shown in Fig. 5. Both the focal length and distance from the transducer to the reflector were $45 \mathrm{~mm}$. The ambient temperature and relative humidity were kept at $20 \pm 3{ }^{\circ} \mathrm{C}$ and $40 \pm 3 \%$, respectively. Typical sound pressure levels were tuned between 155 and $159 \mathrm{~dB}$ for ensuring stable levitation of the droplet. The sound pressure was measured a probe microphone (Bryel \& Kjaer, Type 4138, Diameter: 1/8 inch) for the quantitative evaluation of the sound field. The microphone was fixed on the traverse device, which could move along the $x, y$, and $z$ directions. The behavior of levitated droplets was observed by back-light illumination from the side and coaxial declination from the bottom via a high-speed video camera (Photron Co., Ltd. FASTCAM-Mini UX100) (Fig. 5a). The state inside the droplet was observed with a Nd:YAG sheet laser with a wavelength of $532 \mathrm{~nm}$ (Japan Laser Co., DPGL-5W-L) irradiated from the side of the droplets (Fig. 5b). Fluorescent particles (EBM Co., FLUOSTAR ${ }^{\circledR}$ ) were used for the observation of internal flow, and a fluorescent dye (Kanto Kagaku Co., Rhodamine 6G) was used to observe the mixing behavior. To remove scattered light, a long-pass filter (Kenko Tokina Co., YA3) was used. Figure 5 c shows the signal waveform applied to the transducers when applying oscillation to droplets. The voltage applied to the transducers was modulated from 0 to 1 square wave. Table 1 lists the test fluids and their physical properties. Three fluids of varying density, surface tension and viscosity were selected.

Statistical analysis. The error of the droplet diameter could be a maximum of $<5 \%$ because the levitated droplets were observed with a spatial resolution of $10 \pm 5 \mu \mathrm{m} /$ pix. Sound pressure was measured 3 times, and the error was a maximum of $<7 \%$.

\section{References}

1. Brandt, E. H. Acoustic Physics: Suspended by sound. Nature 413, 474-475 (2001).

2. Foresti, D., Nabavi, M. \& Poulikakos, D. On the acoustic levitation stability behaviour of spherical and ellipsoidal particles. J. Fluid Mech. 709, 581-592 (2012).

3. Foresti, D., Sambatakakis, Simone Bottan, S. \& Poulikakos, D. Morphing surfaces enable acoustophoretic contactless transport of ultrahigh-density matter in air. Sci. Rep. 3, 3176 (2013).

4. Hong, Z. Y. et al. Dynamics of levitated objects in acoustic vortex fields. Sci. Rep. 7, 7093 (2017).

5. Santesson, S. \& Nilsson, S. Airborne chemistry: Acoustic levitation in chemical analysis. Anal. Bioanal. Chem. 378, 1704-1709 (2004).

6. Priego-Capote, F. \& de Castro, L. Ultrasound-assisted levitation: Lab-on-a-drop. Trends in Analytical Chemistry 25, 856-867 (2006).

7. Puskar, L. et al. Raman acoustic levitation spectroscopy of red blood cells and Plasmodium falciparum trophozoites. Lab on a Chip 7, 1125-1131 (2007).

8. Bouyer, C. et al. A Bio-Acoustic Levitational (BAL) assembly method for engineering of multilayered, 3D brain-like constructs, using human embryonic stem cell derived Neuro-Progenitors. Adv. Matter 28, 161-167 (2016).

9. Vasileiou, T., Foresti, D., Bayram, A., Poulikakos, P. \& Ferrari, A. Toward contactless biology: Acoustophoretic DNA transfection. Sci. Rep. 6, 20023 (2016).

10. Tsujino, S. \& Tomizaki, T. Ultrasonic acoustic levitation for fast frame rate X-ray protein crystallography at room temperature. Sci. Rep. 6, 25558 (2016).

11. Foresti, D., Nabavi, M., Klingauf, M., Ferrari, A. \& Polikakos, D. Acoustophoretic contactless transport and handling of matter in air. Proc. Natl. Acad. Sci. USA 110, 12549-12554 (2013).

12. Ochiai, Y., Hoshi, T. \& Rekimoto, J. Three-Dimensional Mid-Air Acoustic Manipulation by Ultrasonic Phased Arrays. PLOS ONE 9, e97590 (2014).

13. Marzo, A. et al. Holographic acoustic elements for manipulation of levitated objects. Nat. Commun. 6, 8661 (2015).

14. Trinh, E. H. \& Robey, J. L. Experimental study of streaming flows associated with ultrasonic levitators. Phys. Fluids 6, 3567-3579 (1994).

15. Yamamoto, Y., Abe, Y., Fujiwara, A., Hasegawa, K. \& Aoki, K. Internal flow of acoustically levitated droplet. Microgravity Sci. Technol. 20, 277-280 (2008).

16. Goda, A., Hasegawa, K., Kaneko, A., Kanagawa, T. \& Abe, Y. External flow structure and interfacial transport phenomena of an acoustically levitated droplet. Jpn J. Multiphase Flow 28, 539-546 (2015).

17. Niwa, M., Watanabe, A., Hasegawa, K., Kaneko, A. \& Abe, Y. Stereo measurement of internal flow structure in volatile droplets. Jpn J. Multiphase Flow 30, 537-546 (2017)

18. Hasegawa, K., Abe, Y. \& Goda, A. Microlayered flow structure around an acoustically levitated droplet under a phase-change process. npj Microgravity 2, 16004 (2016). 
19. Lee, C. P., Anilkumar, A. V. \& Wang, T. G. Static shape and instability of an acoustically levitated liquid drop. Physics of Fluids A 3 , 2497-2515 (1991).

20. Anilkumar, A. V., Lee, C. P. \& Wang, T. G. Stability of an acoustically levitated and flattened drop: An experimental study. Physics of Fluids A 5, 2763-2774 (1993).

21. Tian, Y., Holt, G. \& Apfel, R. E. Deformation and location of an acoustically levitated liquid drop. J. Acoust. Soc. Am. 93, 3096-3104 (1993).

22. Abe, Y., Yamamoto, Y., Hyuga, D., Aoki, K. \& Fujiwara, A. Interfacial Stability and Internal Flow of a Levitated Droplet. Microgravity Sci. Technol 19, 33-34 (2007).

23. Zarembo, L. K. Acoustic streaming in High-intensity ultrasonic fields, editied by L. D. Rozenberg, Plenum, Part III, 137-199 (1971).

24. Danilov, S. \& Mironov, M. Breakup of a droplet in a high-intensity sound fields. J. Acoust. Soc. Am. 92, 2747-2755 (1992).

25. Ahmad, R., Kundu, T. \& Placko, D. Modeling of phased array transducers. J. Acoust. Soc. Am. 117, 1762-1776 (2005).

26. Wada, Y., Yuge, K., Nakamura, R., Tanaka, H. \& Nakamura, K. Dynamic analysis of ultrasonically levitated droplet with moving particle semi-implicit and distributed point source method. Jpn. J. Appl. Phys. 54, 07HE04 (2015).

27. Hoshi, T., Ochiai, Y. \& Reimoto, J. Three-dimensional noncontact manipulation by opposite ultrasonic phased arrays. Jpn. J. Appl. Phys. 53, 07KE07 (2014).

28. Kono, M., Hoshi, T. \& Kakehi, Y. lapillus bug: Creature-like Behaving Particles Based on Interactive Mid-Air Acoustic Manipulation, ACE '14 Proceedings of the 11th Conference on Advances in Computer Entertainment Technology 34 (2014).

29. Gor'kov, L. P. On the forces acting on a small particle in an acoustical field in an ideal fluid. Soviet Physics. Doklady 6, 773-775 (1962).

30. Shen, C. L., Xie, W. J. \& Wei, B. Parametrically excited sectorial oscillation of liquid drops floating in ultrasound. Phys. Rev. E 81, 046305 (2010).

31. Rayleigh, L. On the capillary phenomena of jets. Proc. R. Soc. Lond. 29, 71-97 (1879).

32. Carroll, B. \& Hidrovo, C. Droplet collision mixing diagnostics using single fluorophore LIF. Experiments in Fluids 53, 1301-1316 (2012).

33. Culbertson, C. T., Jacobson, S. C. \& Ramsey, J. M. Diffusion coefficient measurements in microfluidic devices. Talanta 45, 365-373 (2002).

34. Ottino, J. M. The kinematic of mixing: stretching, chaos, and transport $55^{\text {th }}$ ed. (Cambridge University Press, 1989).

35. Weast, R. C. (ed.) CRC Handbook of Chemistry and Physics $55^{\text {th }}$ ed., (CRC Press, 1974).

\section{Acknowledgements}

This work has been supported by JSPS KAKENHI Grant number 15H03925. We thank Prof. Katsuhiro Nishinari, Prof. Tadashi Watanabe, Dr. Satoshi Matsumoto, Dr. Hiroyuki Kitahata, Dr. Akiko Kaneko, Mr. Soma Watahiki, Mr. Atsushi Goda, and Mr. Motonori Niwa for the insightful comments and suggestions. The authors acknowledge support from Japan Aerospace Exploration Agency (JAXA).

\section{Author Contributions}

Y.A. and K.H. designed research. A.W. performed all experiments and analyzed data. All authors worked in preparing the paper.

\section{Additional Information}

Supplementary information accompanies this paper at https://doi.org/10.1038/s41598-018-28451-5.

Competing Interests: The authors declare no competing interests.

Publisher's note: Springer Nature remains neutral with regard to jurisdictional claims in published maps and institutional affiliations.

Open Access This article is licensed under a Creative Commons Attribution 4.0 International License, which permits use, sharing, adaptation, distribution and reproduction in any medium or format, as long as you give appropriate credit to the original author(s) and the source, provide a link to the Creative Commons license, and indicate if changes were made. The images or other third party material in this article are included in the article's Creative Commons license, unless indicated otherwise in a credit line to the material. If material is not included in the article's Creative Commons license and your intended use is not permitted by statutory regulation or exceeds the permitted use, you will need to obtain permission directly from the copyright holder. To view a copy of this license, visit http://creativecommons.org/licenses/by/4.0/.

(C) The Author(s) 2018 